\title{
Stomach Disorder
}

National Cancer Institute

\section{Source}

National Cancer Institute. Stomach Disorder. NCI Thesaurus. Code C26886.

A non-neoplastic or neoplastic disorder that affects the stomach. Representative examples of non-neoplastic disorders include gastritis and gastric ulcer. Representative examples of neoplastic disorders include adenomas, carcinomas, and lymphomas. 